\title{
Calculation of a radially nonhomogeneous ring loaded with normal and tangential loads
}

\author{
Vladimir Andreev ${ }^{\mathrm{a}^{*}}$ and Vladislav Nosyrin ${ }^{\mathrm{b}}$ \\ Moscow State University of Civil Engineering (National Research University), 129337, Moscow, \\ Russia
}

\begin{abstract}
In a two-dimensional plane problem of the theory of elasticity in polar coordinates for a nonhomogeneous body, it is possible to obtain analytical or numerical-analytical solutions only for a one-dimensional nonuniformity [1 - 3]. As a rule, this is possible when all unknown functions depend on the radius. These tasks correspond to the majority of ring and cylindrical structures exposed to the axisymmetric effects of temperature, radiation, humidity, etc. The article considers the problem when radial and ring loads are unevenly distributed along the outer surface of the ring. The problem is solved by two methods: analytical and numerical-analytical, the methods comparison is presented.
\end{abstract}

\section{Introduction}

The article deals with a plane problem of the theory of elasticity in polar coordinates for a radially nonhomogeneous disk under the action of alternating normal and tangential loads. Thus, the problem is two-dimensional with one-dimensional nonuniformity. The variables separation method is used, which is based on the development of the J. Michell generalized solution for the plane problem in polar coordinates. Two solutions of the problem are given: analytical and numerical-analytical, a comparison of two presented calculations results is shown.

\section{The problem statement}

We consider the problem of the equilibrium of a thin ring when forces are applied to its outer surface

$$
p=p_{0}(1+\cos 2 \theta) / 2 ; q=p_{0} \sin 2 \theta / 2,
$$

and the inner surface is free from load (Figure 1).

\footnotetext{
*Corresponding author: asv@mgsu.ru
} 


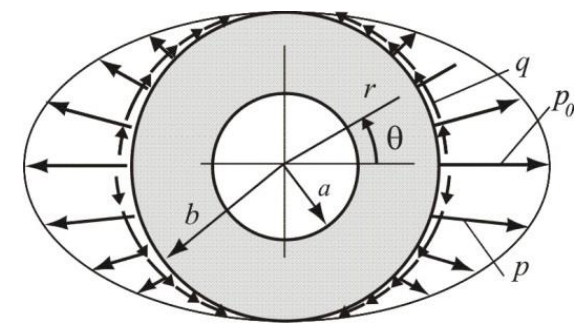

Fig. 1. Design scheme of the problem

The elastic modulus of the ring material is changed as a power function

$$
E(r)=E_{0}\left(\frac{r}{a}\right)^{\delta}
$$

Poisson's ratio: $v=$ const.

\section{Analytical solution}

The analytical solution is based on the method [3] based on the generalized Michell solution (J. H. Michell) development [4] for calculating a plane problem in polar coordinates for heterogeneous designs. In this method, the solution is sought in the form:

$$
\begin{aligned}
& \left(\begin{array}{l}
u \\
v
\end{array}\right)=\left(\begin{array}{l}
\varphi_{0} \\
\psi_{0}
\end{array}\right)+\left(\begin{array}{l}
\varphi_{1} \\
\psi_{1}
\end{array}\right) \cdot \theta+\left(\begin{array}{c}
\varphi_{2} \\
\psi_{2}
\end{array}\right) \cdot \theta \sin \theta+\left(\begin{array}{c}
\varphi_{3} \\
\psi_{3}
\end{array}\right) \cdot \theta \cos \theta+ \\
& +\left(\begin{array}{l}
\varphi_{4} \\
\psi_{4}
\end{array}\right) \cdot \sin \theta+\left(\begin{array}{c}
\varphi_{5} \\
\psi_{5}
\end{array}\right) \cdot \cos \theta+\sum_{n=2}^{\infty}\left[\left(\begin{array}{c}
\varphi_{s n} \\
\psi_{s n}
\end{array}\right) \sin n \theta+\left(\begin{array}{c}
\varphi_{c n} \\
\psi_{c n}
\end{array}\right) \cos n \theta\right]
\end{aligned}
$$

in which each term consists of the product of unknown functions $\left(\varphi_{i}, \psi_{i}\right)$, multiplied by a specific trigonometric function. In (3) indices $s$ and $c$ mean that the corresponding functions are multiplied by $\sin n \theta$ or $\cos n \theta$

The solution is reduced to an infinite partially decaying equations system of the secondorder. In the problem under consideration, the corresponding equations are selected from this system depending on the boundary conditions and formulas for stresses.

In accordance with (1), the boundary conditions have the form:

$$
\begin{aligned}
& r=a, \quad \sigma_{r}=\tau_{r \theta}=0 ; \\
& r=b, \quad \sigma_{r}=p(\theta), \quad \tau_{r \theta}=-q(\theta) .
\end{aligned}
$$

Comparing the boundary conditions and stresses, it can be assumed that the expressions for stresses should contain the functions $\varphi_{0}, \psi_{1}, \varphi_{c 2}$ and $\psi_{s 2}$. From the system of the mentioned differential equations to determine the indicated functions, it is necessary to consider the following four equations:

$$
\left(\lambda^{*}+2 \mu\right)\left(\varphi_{0}^{\prime \prime}+\frac{\varphi_{0}^{\prime}}{r}-\frac{\varphi_{0}}{r^{2}}\right)+\frac{\lambda^{*}+\mu}{r} \psi_{1}^{\prime}-\frac{\lambda^{*}+3 \mu}{r^{2}} \psi_{1}+\left[\left(\lambda^{*}\right)^{\prime}+2 \mu^{\prime}\right] \varphi_{0}^{\prime}+\frac{\left(\lambda^{*}\right)^{\prime}}{r}\left(\varphi_{0}+\psi_{1}\right)=0
$$




$$
\begin{gathered}
\mu\left(\psi_{1}^{\prime \prime}+\frac{\psi_{1}^{\prime}}{r}-\frac{\psi_{1}}{r^{2}}\right)+\mu^{\prime}\left(\psi_{1}^{\prime}-\frac{\psi_{1}}{r}\right)=0 \\
-2 \frac{\lambda^{*}+\mu}{r} \varphi_{c 2}^{\prime}-2 \frac{\lambda^{*}+3 \mu}{r^{2}} \varphi_{c 2}+\mu\left(\psi_{s 2}^{\prime \prime}+\frac{\psi_{s 2}^{\prime}}{r}-\frac{\psi_{s 2}}{r^{2}}\right)-4 \frac{\lambda^{*}+2 \mu}{r^{2}} \psi_{s 2}- \\
-\frac{\mu^{\prime}}{r}\left(2 \varphi_{c 2}-r \psi_{s 2}^{\prime}+\psi_{s 2}\right)=0 \\
\left(\lambda^{*}+2 \mu\right)\left(\varphi_{c 2}^{\prime \prime}+\frac{\varphi_{c 2}^{\prime}}{r}-\frac{\varphi_{c 2}}{r^{2}}\right)-\frac{4 \mu}{r^{2}} \varphi_{c 2}+2 \frac{\lambda^{*}+\mu}{r} \psi_{s 2}^{\prime}-2 \frac{\lambda^{*}+3 \mu}{r^{2}} \psi_{s 2}+ \\
+\left[\left(\lambda^{*}\right)^{\prime}+2 \mu^{\prime}\right] \varphi_{c 2}^{\prime}+\frac{\left(\lambda^{*}\right)^{\prime}}{r}\left(\varphi_{c 2}+2 \psi_{s 2}\right)=0
\end{gathered}
$$

In the above-mentioned equations, a transition is made from a plane deformed state to a plane stressed state by replacing the parameter $\lambda$ to $\lambda^{*}=\frac{E v}{1-v^{2}}$.

Taking into account that the solution of this problem, with consideration to the uniqueness condition, should not have terms containing $\theta$, it follows from (3) that $\psi_{1}^{\prime}-\frac{\psi_{1}}{r}=0$. Taking this equality into account, the equation (6) takes the form $\psi_{1}^{\prime \prime}=0$. The integral of this equation is a linear function that becomes constant at $r=a, b$. Taking into account that the boundary conditions (4) for $\tau_{r \theta}$ with consideration to (1) do not contain a constant, $\psi_{1}=0$ should be stated. Then the equation (5) is simplified:

$$
\left(\lambda^{*}+2 \mu\right)\left(\varphi_{0}^{\prime \prime}+\frac{\varphi_{0}^{\prime}}{r}-\frac{\varphi_{0}}{r^{2}}\right)+\left[\left(\lambda^{*}\right)^{\prime}+2 \mu^{\prime}\right] \varphi_{0}^{\prime}+\frac{\left(\lambda^{*}\right)^{\prime}}{r} \varphi_{0}=0
$$

And it can be used to define the function $\varphi_{0}$.

Thus, the problem can be divided into two: determination of the axisymmetric component of the solution using the equation (9) and determination from the component equations (7), (8) depending on $\theta$. Substitution of (2) into the indicated equations brings them to the form:

$$
\begin{gathered}
\varphi_{0}^{\prime \prime}+(1+\delta) \frac{\varphi_{0}^{\prime}}{r}+(\delta v-1) \frac{\varphi_{0}}{r^{2}}=0 \\
\psi_{s 2}^{\prime \prime}+(1+\delta) \frac{\psi_{s 2}^{\prime}}{r}-\left(\frac{9-v}{1-v}+\delta\right) \frac{\Psi_{s 2}}{r^{2}}-\frac{2(1+v)}{1-v} \frac{\varphi_{c 2}^{\prime}}{r}-\left(\frac{6-2 v}{1-v}+2 \delta\right) \frac{\varphi_{c 2}}{r^{2}}=0 \\
\varphi_{c 2}^{\prime \prime}+(1+\delta) \frac{\varphi_{c 2}^{\prime}}{r}+(\delta v-3+2 v) \frac{\varphi_{c 2}}{r^{2}}+(1+v) \frac{\psi_{s 2}^{\prime}}{r}-(3-v-2 \delta v) \frac{\psi_{s 2}}{r^{2}}=0
\end{gathered}
$$

The solution to the equation (10) is the function [5]:

$$
\varphi_{0}=C_{1} r^{\frac{1-\alpha+\beta}{2}}+C_{2} r^{\frac{1-\alpha-\beta}{2}}
$$


where $\alpha=(1+\delta), \beta=\sqrt{(1-\alpha)^{2}-4(\delta v-1)}$.

The integration constants included in (13) can be found from the boundary conditions for axisymmetric external load component:

$$
r=a, \sigma_{r}=0 ; r=b, \sigma_{r}=p_{0} / 2
$$

The system of two ordinary differential equations (11), (12) can be reduced to one fourth-order equation as follows. We express $\psi_{s 2}^{\prime}$ from the equation (11) as a function of $\psi_{s 2}, \varphi_{c 2}$ and its derivatives:

$$
\psi_{s 2}^{\prime}=f_{1}\left(\psi_{s 2}, \varphi_{c 2}^{\prime \prime}, \varphi_{c 2}^{\prime}, \varphi_{c 2}\right)
$$

Differentiating the resulting expression by $\boldsymbol{r}$ and substituting the equation (a) into it, we obtain:

$$
\psi_{s 2}^{\prime \prime}=f_{2}\left(\varphi_{c 2}^{\prime \prime \prime}, \varphi_{c 2}^{\prime \prime}, \varphi_{c 2}^{\prime}, \varphi_{c 2}, \psi_{s 2}\right)
$$

Substituting (a) and (b) in (5.40), we find an expression for $\psi_{s 2}$ :

$$
\psi_{s 2}=f_{3}\left(\varphi_{c 2}^{\prime \prime \prime}, \varphi_{c 2}^{\prime \prime}, \varphi_{c 2}^{\prime}, \varphi_{c 2}\right)
$$

Differentiating the last equality once with respect to $r$, we equate the resulting expression to the ratio (a), substituting (c) into it. As a result, we obtain a fourth-order equation for the function $\varphi_{c 2}$ :

$$
\begin{aligned}
& r^{4} \varphi_{c 2}^{I V}+(6+2 \delta) r^{3} \varphi_{c 2}^{\prime \prime \prime}+\left(5 \delta+\delta^{2}+v \delta-3\right) r^{2} \varphi_{c 2}^{\prime \prime}+\left(v \delta^{2}+v \delta-9 \delta-9\right) r \varphi_{c 2}^{\prime}+ \\
& +\left(3 v \delta^{2}+3 v \delta-3 \delta+9\right) \varphi_{c 2}=0
\end{aligned}
$$

The resulting equation can be reduced to a differential equation with constant coefficients by introducing the variable $t$ using the linear connection $r=e^{t}$ :

$$
\begin{aligned}
& \frac{d^{4} \varphi_{c 2}}{d t^{4}}+2 \delta \frac{d^{3} \varphi_{c 2}}{d t^{3}}+\left(\delta^{2}+v \delta-\delta-10\right) \frac{d^{2} \varphi_{c 2}}{d t^{2}}+\left(v \delta^{2}-\delta^{2}-10 \delta\right) \frac{d \varphi_{c 2}}{d t}+ \\
& +\left(3 v \delta^{2}+3 v \delta-3 \delta+9\right) \varphi_{c 2}=0 .
\end{aligned}
$$

The characteristic equation corresponding to the obtained equation will be

$$
l^{4}+2 \delta l^{3}+\left(\delta^{2}+v \delta--10\right) l^{2}+\left(v \delta^{2}-\delta^{2}-10 \delta\right) l+3 v \delta^{2}+3 v \delta-3 \delta+9=0 .
$$

Using the replacement $\eta=l^{2}+\delta l$, this equation can be reduced to a square equation:

$$
\eta^{2}+(v \delta-\delta-10) \eta+3 v \delta^{2}+3 v \delta-3 \delta+9=0
$$


The final solution to the equation (14) is represented as:

$$
\varphi_{c 2}=\sum_{i=1}^{4} D_{i} e^{l t},
$$

which constants $D_{i}$ are determined from the boundary conditions for the axially asymmetric

component:

$r=a, \sigma_{r}=\tau_{r \theta}=0 ; r=b, \sigma_{r}=p_{0} \cos 2 \theta / 2, \tau_{r \theta}=-p_{0} \sin 2 \theta / 2$.

The function $\psi_{c 2}$ can be found from the equation (c).

Below is an example of the calculation carried out for the following input data: $\delta=-1$; $b / a=2 ; v=1 / 3 ; E=2 \cdot 10^{4} \mathrm{MPa}$

Figure 2 shows the stress plots $\sigma_{\theta}$ along three directions of the radius. From the graphs presented, we can conclude that taking into account the heterogeneity in this case does not lead to a qualitative change in the nature of the diagrams. As for the numerical differences, in some cases they amount to about $20 \%$. It is natural to assume that with a more significant inhomogeneity, the differences in the results for homogeneous and nonhomogeneous materials can be more significant.

In this task, it is enough to simply trace the dependence of the displacements on material inhomogeneity. Fig. 3 shows the points displacements diagrams of the inner contour of the ring along the angular coordinate. It can be seen that the displacement in a non-uniform ring is greater than in a homogeneous one. This fact is explained by the fact that at $\delta=-1$ the modulus of elasticity decreases from the inner contour to the outer one by half, and this leads to a decrease in the rigidity of the ring as a whole.

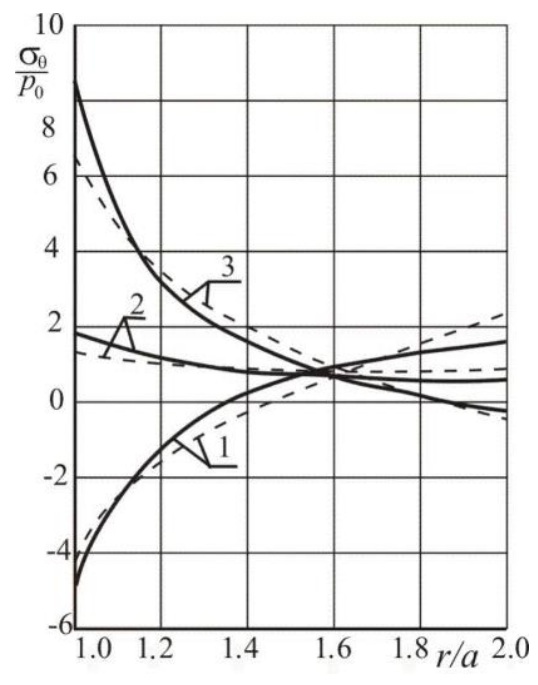

Fig. 2.Voltage $\sigma_{\theta}$ in the ring:

$1-\theta=0,2-\theta=\pi / 4,3-\theta=\pi / 2$;

heterogeneous material,

- - - - homogeneous material

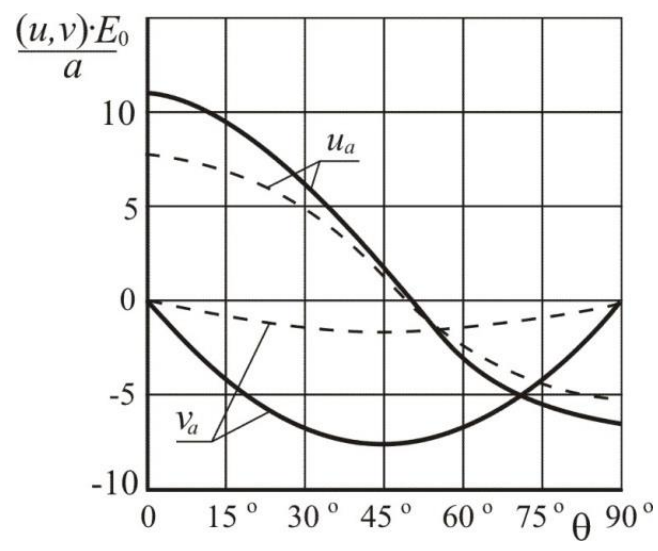

Fig. 3. Moving points of the inner contour of the ring: heterogeneous material,

- - - homogeneous material 


\section{Solution by numerical-analytical method}

In this section, we consider the problem, the analytical solution of which was obtained in section 3. Using this problem as an example, the application of the numerical-analytical method will be demonstrated, including the numerical solution of the ordinary differential equations systems $[6,7$, etc.]. At the same time, it is possible to determine the accuracy of the method under consideration.

As shown above, in order to satisfy the boundary conditions (4) taking into account (1), it is sufficient in the formulas (3) to be limited by the terms $\varphi_{0}, \varphi_{c 2} \cos 2 \theta$ and $\psi_{s 2} \sin 2 \theta$. Expressions for the stresses $\sigma_{r}$ and $\tau_{r \theta}$, included in the boundary conditions will have the form:

$$
\begin{gathered}
\sigma_{r}=\frac{E}{(1+v)(1-v)}\left[\left(\varphi_{0}^{\prime}+v \frac{\varphi_{0}}{r}\right)+\left(\varphi_{c 2}^{\prime}+v \frac{\varphi_{c 2}+2 \psi_{s 2}}{r}\right) \cdot \cos 2 \theta\right] \\
\tau_{r \theta}=\frac{E}{2(1+v)}\left(\psi_{s 2}^{\prime}-\frac{\psi_{s 2}+2 \varphi_{c 2}}{r}\right) \cdot \sin 2 \theta
\end{gathered}
$$

For the functions $\varphi_{0}, \varphi_{c 2}$ and $\psi_{s 2}$ at the law of change in the modulus of elasticity (2), the following equations are valid (10) - (12).

Let us reduce the equation (10) to a system of two equations of the first order, introducing the notation $y_{1}=\varphi_{0}, y_{2}=\varphi_{0}^{\prime}$ :

$$
y_{1}^{\prime}=y_{2} ; \quad y_{2}^{\prime}=-(1+\delta) \frac{y_{2}}{r}-(\delta v-1) \frac{y_{1}}{r^{2}} \text {. }
$$

The boundary conditions (4) for the axisymmetric component of stresses will take the form:

$$
r=a, \quad y_{2}+v \frac{y_{1}}{r}=0 ; \quad r=b, y_{2}+v \frac{y_{1}}{r}=-\frac{p_{0}(1+v)(1-v)}{2 E(b)} .
$$

The equations (11), (12) with the introduction of the notation $y_{1}=\varphi_{c 2}, y_{2}=\varphi_{c 2}^{\prime}$, $y_{3}=\psi_{s 2}, \quad y_{4}=\psi_{s 2}^{\prime}$ are reduced to a system of four equations of the first order form. Adding to these equations the boundary conditions (4) for axially asymmetric component, we obtain a boundary value problem for the functions $\varphi_{c 2}$ and $\psi_{s 2}$.

The calculation was carried out on the interval $(a, b)$ on a uniform one when partitioned into 100 steps for the same initial data as in the section 3. Table 1 shows the comparative values of stresses in a nonhomogeneous ring at $\theta=45^{\circ}$, obtained as a result of the analytical and numerical calculations.

Table 1. Ring stresses

\begin{tabular}{|c|c|c|c|c|}
\hline \multirow{2}{*}{$r / a$} & \multicolumn{2}{|c|}{$\begin{array}{c}\text { Analytical } \\
\text { solution }\end{array}$} & \multicolumn{2}{c|}{$\begin{array}{c}\text { Numerical } \\
\text { solution }\end{array}$} \\
\cline { 2 - 5 } & $\sigma_{r}$ & $\tau_{r \theta}$ & $\sigma_{r}$ & $\tau_{r \theta}$ \\
\hline 1.0 & 0 & 0 & 0 & 0 \\
\hline
\end{tabular}




\begin{tabular}{|l|l|l|l|l|}
\hline 1.2 & 0.252 & -1.266 & 0.263 & -1.296 \\
\hline 1.4 & 0.377 & -1.338 & 0.378 & -1.363 \\
\hline 1.6 & 0.444 & -1.108 & 0.444 & -1.121 \\
\hline 1.8 & 0.480 & -0.807 & 0.480 & -0.813 \\
\hline 2.0 & 2.000 & -0.500 & 2.000 & -0.500 \\
\hline
\end{tabular}

Comparison of the results obtained by the two methods give a possibility to conclude that the numerical-analytical method is quite accurate.

If we consider a ring, the ratio of the outer and inner radii of which is sufficiently large, then on the basis of the considered calculation method, we can obtain a solution to the tension - compression problem of a plate with a small circular hole. In [8], the solutions for the problems of stretching a nonhomogeneous plate with a hole in one direction, tension compression in two directions, and shear of a plate with a hole were obtained. Based on the numerical-analytical calculation of a homogeneous plate and comparing the results with the solution of the Kirsch problem [4], it was shown that satisfactory accuracy is achieved when the ratio of the plate size to the hole radius is greater than 10 .

\section{Conclusion}

The problem considered in the article is an example of using a generalized method for calculating a plane two-dimensional problem for a radially nonhomogeneous ring. The possibility of obtaining an analytical solution to such problems largely depends on the material heterogeneity, i.e., primarily on the dependence of the modulus of elasticity of the material on the radius. The power-law dependence of the elastic modulus on the radius selected in the article is the simplest.

The obtained second solution by the numerical method shows good compatibility with the analytical solution and can be used for solving two-dimensional plane problems with radial inhomogeneity for any continuous dependence of the elastic modulus on the radius.

\section{References}

1. V.I. Andreev, Applied Mechanics 23 (4), 16-23 (1987).

2. V.I. Andreev, Some problems and methods of mechanics of nonhomogeneous bodies (Moscow, ASB, 2002).

3. V.I. Andreev, N.Yu. Tsybin, WIT Transactions on Modeling and Simulation 57, 215227 (2014)

4. S.P. Timoshenko, J.N. Goodier, Elasticity theory (Moscow, Nauka, 1979).

5. E. Kamke, Ordinary Differential Equations Handbook (Moscow, Nauka, 1976).

6. A.A. Samarskiy, A.V. Gulin, Numerical Methods (Moscow, Nauka, 1989, 428).

7. V.N. Kukudjhanov, Numerical Methods in Continuum Mechanics (Moscow, Moscow State Aviation technological University, 2006).

8. V.I. Andreev, K.A. Kerimov, A.V. Smolov, Strength and Structural Theory 53, 62-67 (1989). 